\title{
Biological and haematological safety profile of oral amodiaquine and chloroquine in healthy volunteers with or without Plasmodium falciparum infection in northeast Tanzania
}

\author{
J.J. MASSAGA ${ }^{1 *}$, J.P. LUSINGU'², R. MAKUNDE ${ }^{3}$ H.M. MALEBO ${ }^{6}$, M.M. CHILE ${ }^{2}$, J.A. AKIDA ${ }^{2}$, M.M. \\ LEMNGE $^{2}$, A.M. RØNN ${ }^{4}$, T.G. THEANDER ${ }^{5}$, I.C. BYGBJERG ${ }^{4}$ and A.Y. KITUA ${ }^{6}$ \\ ${ }^{1}$ Centre for Enhancement of Effective Malaria Interventions, P.O. Box 9653, Dar es Salaam, Tanzania \\ ${ }^{2}$ Tanga Medical Research Centre, Tanga, Tanzania \\ ${ }^{3}$ Bombo Regional Hospital, Tanga, Tanzania \\ ${ }_{4}^{4}$ Institute of Public Health, University of Copenhagen, Copenhagen, Denmark \\ ${ }^{5}$ Centre for Medical Parasitology, University of Copenhagen, Copenhagen, Denmark \\ ${ }^{6}$ National Institute for Medical Research, Headquarters, Dar es Salaam, Tanzania
}

\begin{abstract}
Amodiaquine (AQ), an effective antimalarial drug for uncomplicated malaria, has been greatly restricted after cases of life-threatening agranulocytosis and hepatic toxicity during prophylactic use. We conducted a hospital based open-label randomised clinical trial in 40 indigenous semi-immune healthy adult male volunteers with and without malaria parasites. The objective was to collect data on biological and haematological safety, tolerability, and parasitological efficacy to serve as baseline in the evaluation of the effectiveness of AQ preventive intermittent treatment against malaria morbidity in infants. Volunteers were stratified according to parasitaemia status and randomly assigned 20 participants each arm to three days treatment with either AQ or chloroquine (CQ). The level of difference of selected haematological and hepatological values pre-and post-trial were marginal and within the normal limits. Clinical adverse effects mostly mild and transient were noticed in 33.3\% CQ treated-aparasitaemic, $23.8 \%$ of CQ treated-parasitaemic, $28.6 \%$ of AQ-treated parasitaemic and $14.3 \%$ of aparasitaemic receiving AQ. Amodiaquine attained $100 \%$ parasitological clearance rate versus $70 \%$ in CQ-treated volunteers. The findings indicate that there was no agranulocytosis or hepatic toxicity suggesting that AQ may pose no public health risk in its wide therapeutic dosage uses. Larger studies are needed to exclude rare adverse effects.
\end{abstract}

Key words: Amodiaquine, chloroquine, biological, haematological, safety, treatment, malaria, Tanzania

\section{Introduction}

Amodiaquine (AQ) is currently among potent blood schizonticide 4-aminoquinoline drugs available for treatment of uncomplicated malaria (Olliaro et al., 1996), and is a possible candidate for preventive intermittent treatment. Preventive intermittent treatment is a recent strategy that is considered an opportune and cost-effective control measure for combating malaria in infants in malaria endemic countries (WHO, 1998).

Since its discovery in 1940's (Greenwood, 1995), AQ established a good safety reputation and was used extensively for chemotherapy in malaria as an alternative to chloroquine (CQ) or to treat CQ failures as well as for chemoprophylaxis in non-immune travellers visiting malarious endemic countries (Hatton et al., 1986). Amodiaquine when used for prophylaxis in non-immune travellers may induce toxic hepatic and potential lethal agranulocytosis (Hatton et al., 1986, Larrey et al., 1986, CDC, 1986). However, chemotherapeutic studies have uniformly indicated that $\mathrm{AQ}$ when given in doses required for treatment is efficacious both without serious haematological life threatening adverse effects (Mengesha \& Makonne, 1999), and with no potential biological toxicity associated with liver or kidney functions (Brasseur et al., 1999).

The risk of severe hepatic disorders and agranulocytosis to AQ curtailed its use for chemoprophylaxis and chemotherapy (WHO, 1990), but a systematic review doubted about toxicity for chemotherapeutic use (Olliaro et al., 1996). Accordingly, Nevill et al. (1994) suggested that the restriction to contraindicate AQ for chemotherapy was decided prematurely: published, peer-reviewed data unquestionably verifying its toxicity in chemotherapeutic dosage were lacking. This is strongly supported by Breckenridge \& Winstanley, (1997) who emphasize that AQ in therapeutic doses is usually free from toxic effects.

Despite considerable ample body of literature on efficacy and safety in treating uncomplicated falciparum

\footnotetext{
* Correspondence: Dr. Julius J. Massaga; E-mail: jjmassaga@gmail.com
} 
malaria in African children (Nevill et al., 1994, Brasseur et al., 1999, Mengesha \& Makonne, 1999), there is dearth of data on safety of AQ in healthy Tanzanian population. Since AQ will be administered to apparently healthy infants in attempt to protect them against malaria morbidity, a careful risk-benefit assessment is required to ensure that the toxicity of AQ does not outweigh the risk of acquiring malaria. The pharmacokinetics profile of orally administered AQ in malaria patients and healthy volunteers has shown to be similar (Krishna \& White, 1996); but in a recent pharmacological study of AQ combined with artesunate undertaken in South Africa, showed increased levels of liver transaminases in a normal volunteer (Orrell et al., 2001). Therefore, we conducted a hospital based open-label randomised clinical trial to evaluate safety, tolerability and efficacy of AQ in semi-immune indigenous adult healthy volunteers with and without Plasmodium falciparum infection in Tanzania. These results were valuable before a study of preventive intermittent treatment with AQ in infants (Massaga et al., 2003).

\section{Material and Methods}

\section{Study subjects}

Healthy male volunteers were recruited as part of a study on preventive intermittent treatment in infants. Volunteers were judged to be healthy on the basis of standard haematological and biochemical tests as well as full history and complete medical examinations. Volunteers also were required to be aged between 15 and 45 years, malaria parasite negative or positive asymptomatic, no history of fever, free from medication including intake of antimalarials for the past four weeks, and without history of congenital abnormalities or chronic and severe diseases. Volunteers were excluded if they had contraindication for the use of test drugs especially previous history of sore throat with AQ.

\section{Study design}

This study was an open-label randomised clinical trial in which volunteers received either AQ or CQ. Volunteers were stratified into two groups of malaria parasite positive and malaria parasite negative. In each stratum randomisation lists were produced, and volunteers were individually randomised to receive either $\mathrm{AQ}$ (treatment) or CQ (control). An individual that was unconnected with the study did randomisation and drug administration. Volunteers were weighed and evenly received oral standard recommended doses (25mg base/kg body weight for consecutive three days) of either AQ or CQ and all drug administration were supervised and observed for 30 minutes. Both AQ (200mg base) and
CQ (150mg base) were obtained from a pharmaceutical supplier in Tanga, Tanzania and the drugs were product from Nemi Pharma Prt Ltd, Mumbai, India.

Drug administration was done in the evening after a light meal, and time of dosing for each volunteer was recorded. There were no concomitant medications or non-drug therapy taken or administered during the study. Consumption of alcoholic beverages were avoided a day before taking the test drug and during the 8 days of the study. The study was single blind in that clinicians conducting clinical evaluation and technologists were not informed of the group allocation. The study was carried out at Bombo Tanga Regional Hospital where volunteers were hospitalised for eight days to allow close monitoring of side effects and regularly followed till day 15 after commencing treatment.

\section{Assessment of biochemical, haematological and clini- cal adverse effects}

Five $\mathrm{ml}$ of venous blood were collected by venepuncture into tubes containing EDTA at days 0, 3 and 7 for biochemical tests, parasitaemia and haematological indices. Biochemical tests included serum alanine aminotransferase (ALT), asparate aminotransferase (AST), creatinine and total bilirubin. Blood smears were immediately stained with Giemsa and read under microscope for malaria parasite identification and enumeration. Total white blood cells and differentials were determined using Neubauer haematocytometer kit (Roth, Karlsruhe, Germany) and microscopic examination of thin blood films, respectively. Packed cell volume (PCV) was measured using a micro-haematocrit centrifuge.

Clinical evaluation was done by one of investigators (JPL) every twelve hours and information was recorded on a standard form. The evaluation included assessment of vital signs (supine blood pressure, pulse and respiration rate, and axillary temperature). Chest and abdomen were also examined. The occurrence of clinical adverse effects, including those volunteered by patients and those elicited by general questioning, and abnormalities in safety laboratory tests during treatment were documented.

\section{Data analysis}

All information was transferred onto a database using Epi Info 6.04b. After cleaning, the file was imported into SPSS 10.0 (SPSS, Chicago, Illinois, USA) statistical package for analysis. Statistical tests used included $\chi^{2}$ for categorical variables and are presented as numbers and percentages. A one-way analysis of variance (ANOVA) and Tukey's honestly significant difference statistical analyses were employed to compare the differences in mean changes between baseline and values at the end of 
the study.

\section{Ethical consideration}

The study protocol was approved by the Medical Research Coordinating Committee of the National Institute for Medical Research, Tanzania. All volunteers gave oral consent to participate in the study following explanation of the aim and conduct of the study.

\section{Results}

\section{Biological characteristics of the subjects}

The trial consisted of 40 male volunteers whose ages ranged from 16 to 45 years (mean age $24.7 \pm 7.41$ years), and all completed the eight days follow-up. Volunteers had no history of significant diseases or a clinical history that was expected to affect the results of the study. At baseline, the four groups were found to be homogenous in demographic, biochemical, clinical and haematological parameters except for parasitaemia status as two groups were malaria parasite negative. Four volunteers had neutrophil counts slightly below the lower limit of which one belonged in parasitaemic group receiving CQ, two and one in parasitaemic and aparasitaemeic groups receiving AQ respectively. Four volunteers had slightly elevated serum total bilirubin three in CQ-treated parasitaemic and one from CQtreated aparasitaemic group (Table 1).

Table 1: Demographic, clinical and biological characteristic of volunteers before treatment with chloroquine or amodiaquine

\begin{tabular}{|c|c|c|c|c|}
\hline \multirow[t]{2}{*}{ Characteristics } & \multicolumn{2}{|c|}{ Chloroquine } & \multicolumn{2}{|c|}{ Amodiaquine } \\
\hline & $\begin{array}{l}\text { Parasite negative } \\
\qquad(\mathrm{n}=10)\end{array}$ & $\begin{array}{c}\text { Parasite positive } \\
\quad(n=10)\end{array}$ & $\begin{array}{l}\text { Parasite negative } \\
\qquad(\mathrm{n}=10)\end{array}$ & $\begin{array}{c}\text { Parasite positive } \\
(\mathbf{n}=10)\end{array}$ \\
\hline \multicolumn{5}{|l|}{ Demography } \\
\hline Age (years \pm SD) & $29.4 \pm 9.28$ & $22.1 \pm 3.73$ & $24.4 \pm 8.33$ & $23.1 \pm 5.78$ \\
\hline Weight (kg \pm SD) & $58.5 \pm 6.47$ & $60.0 \pm 5.10$ & $54.8 \pm 4.24$ & $63.1 \pm 9.12$ \\
\hline Height (cm \pm SD) & $166.6 \pm 6.08$ & $165 \pm 4.82$ & $166.3 \pm 5.64$ & $169 \pm 8.37$ \\
\hline \multicolumn{5}{|l|}{ Vital Signs } \\
\hline $\begin{array}{l}\text { Systolic blood pressure } \\
\text { (mmHg) }\end{array}$ & $119(100-140)$ & $122(100-160)$ & $115(100-110)$ & $113(100-120)$ \\
\hline $\begin{array}{l}\text { Diastolic blood pressure } \\
\text { (mmHg) }\end{array}$ & $75(60-90)$ & $73(60-80)$ & $76(60-90)$ & $73(60-80)$ \\
\hline Pulse rate, beats/min & $73.4(60-88)$ & $70.0(60-80)$ & $70.8(62-86)$ & $69.4(60-80)$ \\
\hline Respiration rate/min & $21.0(18-24)$ & $20.4(18-22)$ & $19.4(18-22)$ & $20.6(18-24)$ \\
\hline Temperature ${ }^{\circ} \mathrm{C}$ & $36.67(36.0-37.3)$ & $36.72(36.1-37.1)$ & $36.52(36.0-37.4)$ & $36.5(36.1-37.2)$ \\
\hline \multicolumn{5}{|l|}{ Laboratory Data (range) } \\
\hline Parasite density, $\mu \mathrm{l}$ & Not applicable & $129(40-520)$ & Not applicable & $239(40-60117)$ \\
\hline ALT, IU/L & $15.5(7.0-38.0)$ & $12.6(6.0-35.0)$ & $14.0(6.0-35.0)$ & $15.1(6.0-35.0)$ \\
\hline AST, IU/L & $17.5(14.0-23.0)$ & $16.3(11.0-27.0)$ & $15.9(13.0-19.0)$ & $16.9(13.0-23.0)$ \\
\hline Creatinine, $\mu \mathrm{mol} / \mathrm{L}$ & $81.2(58.0-109)$ & $76.7(58.0-103.0)$ & $71.4(52.0-104.0)$ & $70.8(54.0$ 86.0) \\
\hline Bilirubin, $\mu \mathrm{mol} / \mathrm{L}$ & $12.3(3.0-24.0)$ & $15.1(7.0-23.0)$ & $13.5(4.0-23.0)$ & $12.8(3.0-20.0)$ \\
\hline $\begin{array}{l}\text { PCV ( } 95 \% \text { confidence } \\
\text { interval) }\end{array}$ & $39.6(37.9-41.3)$ & $38.0(36.5-39.5)$ & $40.2(38.1-42.3)$ & $37.2(36.0-38.4)$ \\
\hline Leucocyte count x $10^{9}$ & $8.7(6.9-10.0)$ & $7.3(4.5-12.0)$ & $8.3(4.4-10.0)$ & $7.0(4.8-8.4)$ \\
\hline Neutrophils & $4.4(3.2-6.0)$ & $3.7(2.1-5.1)$ & $4.2(2.4-4.9)$ & $3.5(2.3-5.2)$ \\
\hline
\end{tabular}




\section{Haematological adverse effects}

There were no significant changes from pre-trial within and between the treatment groups, but five (12.5\%) volunteers had neutrophil counts below the lower limit of $2.5 \times 10^{9}$, of whom two were from malaria parasite negative receiving AQ, two from malaria parasite negative volunteers receiving CQ and one from parasite positive receiving CQ (Table 2). Comparing to pre-trial counts, among those with low counts, the reduction in absolute counts ranged between 0.3 and $0.5 \times 10^{9}$ in $\mathrm{AQ}$ recipients and 0.1 to $0.2 \times 10^{9}$ for those receiving CQ. The paired pre- and post-trial for other cell counts among the four groups were comparable between AQ and CQ treatment groups and were within normal ranges.

Table 2: Biological and haematological characteristics changes at day seven compared to pre-trial.

\begin{tabular}{llllll}
\hline Characteristics & $\begin{array}{l}\text { Chloroquine } \\
\text { Negative }\end{array}$ & Positive & $\begin{array}{l}\text { Amodiaquine } \\
\text { Negative }\end{array}$ & Positive & P-value \\
\hline ALT in IU/L, \pm SD & $1.50 \pm 6.04$ & $2.40 \pm 6.55$ & $0.40 \pm 2.46$ & $(1.10) \pm 3.60$ & 0.439 \\
AST in IU/L & $(2.00) \pm 2.63$ & $(1.40) \pm 2.41$ & $(1.60) \pm 3.20$ & $(3.1) \pm 4.70$ & 0.677 \\
Creatinine in $\mu \mathrm{mol} / \mathrm{L}$ & $3.50 \pm 12.60$ & $(7.00) \pm 19.39$ & $2.70 \pm 21.23$ & $(5.90) \pm 12.71$ & 0.374 \\
Bilirubin in $\mu \mathrm{mol} / \mathrm{L}$ & $(0.20) \pm 6.30$ & $0.40 \pm(4.22)$ & $0.30 \pm 6.72$ & $0.10 \pm 5.38$ & 0.996 \\
Leucocyte count x 109 & $0.86 \pm 1.26$ & $0.76 \pm 1.85$ & $0.13 \pm 1.78$ & $1.33 \pm 1.90$ & 0.783 \\
Neutrophils count x 109 & $0.54 \pm 1.57$ & $0.02 \pm 1.29$ & $0.15 \pm 1.36$ & $0.25 \pm 1.24$ & 0.848 \\
\hline
\end{tabular}

Values in parentheses indicates a decrease in means from the pre-trial values

\section{Biological adverse effects}

The major biological parameters were not significantly altered during the treatment in all four groups (Table 2). Liver function tests showed a slight decrease in AST and an increase ALT values post-trial, but no case had the values below or above the limit values (lower limit for AST 15.0IU/L; upper limit 30.0IU/L for ALT). Total bilirubin level exceeded $20.5 \mu \mathrm{mol} / \mathrm{L}$ in $7(17.5 \%)$ volunteers, of which 3 were malaria parasite positive receiving CQ, 2 from malaria parasite negative receiving CQ and 1 in each group treated with AQ. The level was slightly higher in malaria parasite negative, both those receiving AQ, $7.2 \mu \mathrm{mol} / \mathrm{L}$, and those on CQ 6.5 $\mu \mathrm{mol} / \mathrm{L}$. Renal function tests showed no significant change in plasma creatinine post-trial in all four groups, but one malaria parasite negative volunteer receiving CQ had elevated value on day 3 reaching $136 \mu \mathrm{mol} / \mathrm{l}$ (10 $\mu \mathrm{mol} / \mathrm{L}$ above the normal value). Other biological and haematological values were normal. The value was within normal $(92 \mu \mathrm{mol} / \mathrm{L})$ on day 7 .

\section{Clinical adverse effects}

Twenty-one (52.5\%) volunteers experienced 48 possible adverse effects (Table 3 ). Adverse effects first appeared about $12 \mathrm{hrs}$ after instituting therapy, and most of these were mild, transient requiring no medication and last- ing not more than $12 \mathrm{hrs}$. The adverse reactions rate did not significantly differ among the four groups but were frequently observed with CQ groups $12(57.1 \%)$ versus $A Q$ groups 9 (42.9\%), $P=0.527$. Overall, malaria negative volunteers receiving CQ had experienced more adverse effects comparing to other groups at a rate of $7(33.3 \%)$. The rates of occurrence in other treatment groups were $5(23.8 \%)$ for CQ-treated malaria parasite positive, $6(28.6 \%)$ for AQ-treated malaria positive and $3(14.3 \%)$ for AQ-treated malaria negative group. The reaction observed or reported included gastrointestinal (abdominal) discomfort particularly nausea. Overall, the relative risk (RR) of developing abdominal discomfort with AQ treatment was 0.67 demonstrating statistically insignificant equivalence [ $95 \%$ confidence interval (CI), 0.22, 2.01]. Other reactions included general body malaise and headache. Fever was reported in 5 $(12.5 \%)$ volunteers, but measured temperature in fever complainants ranged between $36.1^{\circ} \mathrm{C}$ and $37.0^{\circ} \mathrm{C}$. There was no trend in blood pressure readings without clinical relevance, but remained within normal range and no other abnormal findings for objective signs including pulse rate and respiration rate were found. 
Table 3: Number (\%) of volunteers for most frequently reported clinical adverse effects related to treatment with chloroquine or amodiaquine

\begin{tabular}{|c|c|c|c|c|c|}
\hline \multirow[b]{2}{*}{ Adverse effects } & \multirow[b]{2}{*}{$\begin{array}{l}\text { All volunteer } \\
\qquad \mathrm{n}=\mathbf{4 0}\end{array}$} & \multicolumn{2}{|c|}{ Chloroquine } & \multicolumn{2}{|c|}{ Amodiaquine } \\
\hline & & $\begin{array}{l}\text { Negative Volun- } \\
\text { teers } n=10\end{array}$ & $\begin{array}{c}\text { Positive Volun- } \\
\text { teers } n=10\end{array}$ & $\begin{array}{l}\text { Negative Volun- } \\
\text { teers } n=10\end{array}$ & $\begin{array}{c}\text { Positive Volun- } \\
\text { teers } n=10\end{array}$ \\
\hline Abdominal discomfort & $10(25.0)$ & $1(10)$ & $5(50)$ & $1(10)$ & $3(30)$ \\
\hline Headache & $8(20.0)$ & $3(30)$ & $2(20)$ & $1(10)$ & $2(20)$ \\
\hline Body Malaise & $5(12.5)$ & $2(20)$ & $2(20)$ & $1(10)$ & $1(10)$ \\
\hline Fever & $5(12.5)$ & $2(20)$ & $1(10)$ & $1(10)$ & $1(10)$ \\
\hline
\end{tabular}

\section{Outcome measures of efficacy}

Overall therapeutic responses among malaria parasite positive groups were good. In AQ group all 10 volunteers responded favourably to treatment. By day 3, only one $(10 \%)$ remained positive, with geometric mean parasite density (GMPD) of 40 asexual parasites per $\mu$ l. A rapid decline in level of parasitaemia was observed in all volunteers. On day 7 of follow-up, all volunteers were free of asexual parasites. In contrast, $4(40 \%)$ in a group receiving $\mathrm{CQ}$, geometric mean parasite density (GMPD) 69 parasites $/ \mu 1$, (range, $40-120$ parasite $/ \mu 1$ ) were parasite positive on day 3 post-dosing. At day 7 , three $(30 \%)$ of the volunteers remained positive and GMPD increased reaching 600 parasites/ $\mu$ l.

All groups experienced increased mean PCV value between pre- and post trial, but the difference was not significant. The values at day 7 were 40.1 (95\% CI, 37.7 - 42.5), 38.7 (95\% CI, 37.5 - 39.8), 41.1 (95\% CI, 39.5 - 42.7) and 39.1 (95\% CI, 38.1 - 40.1) for CQ-malaria negative, CQ-malaria positive, AQ-malaria parasite negative and AQ-malaria parasite positive group, respectively. The largest increase was found in malaria positive which received AQ (1.9\%), followed by malaria negative group receiving AQ $(0.9 \%)$, and malaria positive treated with CQ $(0.7 \%)$. The increase was smallest in malaria negative which received CQ $(0.5 \%)$.

\section{Discussion}

Throughout the entire study, no major serious abnormalities attributable to AQ were observed. There were no abnormal vital signs and no significant changes in values of laboratory biological and haematological tests. The results from this study therefore provide extended safety profile described in a previous chemotherapeutic study (Olliaro et al., 1996). In general, the frequencies of AQ-mediated serious toxicity are exceedingly rare among chemoprophylatic users (Hatton et al. 1986; Phillips-Howard \& West 1990). From epidemiological point of view, large sample size post-marketing surveillance studies would be required to detect such rare incidences. Nevertheless, despite a small sample size in the present study neither agranulocytosis nor liver toxicity fatal case was observed in AQ treated subjects. Similar observations have been reported elsewhere (Breckenridge \& Winstanley, 1997). Full resolution of laboratory abnormalities was observed in all cases of mild abnormalities within seven days post trial. The results from our study offer supportive evidence of the extremely low prospect of toxic adverse reactions that might occur during the wide use of $\mathrm{AQ}$.

Comparison of clinical adverse effects in our study suggests that all treatment regimens were equally well tolerated. The most frequent adverse effect reported was gastrointestinal toxicity (nausea) among CQ recipients. A similar relatively high gastrointestinal toxicity has been observed in malaria patients treated with CQ in central and west Africa (Brasseur et al., 1999). Low gastrointestinal toxicity can be of particular importance for patients receiving chemotherapy, as many chemotherapeutic agents induce nausea and vomiting. Less frequent gastrointestinal adverse drug effects in volunteers receiving $\mathrm{AQ}$ are an important advantage that should be taken into account when preventive intermittent $A Q$ treatment is implemented.

Changes in renal and hepatic biochemical makers were not clinically significant between AQ- and CQtreated volunteers either with or without parasitaemia. The findings are in contrast with recent results where AST and ALT levels were elevated following two doses of AQ combined with artesunate, and AQ was incriminated for the outcome (Orrell et al., 2001). Although total serum bilirubin was elevated, in none of the cases had jaundice (the main characteristic presentation) (Larrey et al., 1986). The level of increase was mild and jaundice clinical presentation was unexpected based upon pre-clinical studies and previous experience with amodiaquine (Greenwood, 1995). A non-serious de- 
cline in neutrophils counts in $12.5 \%$ of volunteers was observed. However, the values were only marginally below the normal range. In agranulocytosis, neutrophils counts should be below than $0.5 \times 10^{9} / 1$ (normal range 2.5-7.7 $\times 10^{9} / \mathrm{l}$ ) while other cell counts are normal (Silverthon, 1998). The absolute number of neutrophils diminished slightly more in the AQ group compared with CQ groups, but variation was mild suggesting that the differences between the groups were not of clinical significance. Our findings are consistent with results from previous chemotherapeutic studies (Nevill et al., 1994; Olliaro et al., 1996; Brasseur et al., 1999; Mengesha \& Makonne, 1999), which demonstrated that AQ given at therapeutic dosage exhibits no life-threatening toxicity. It appears that the reason of increasing risk of life-threatening agranulocytosis and hepatic toxicity during AQ prophylactic administration (Hatton et al., 1986; CDC, 1986; Phillips-Howard \& West 1990) is a result of doseindependent hypersensitivity reactions (Hatton et al., 1986). Besides cell-mediated reaction, it is conceivable that excessive uncontrolled use of AQ (Kennedy, 1955, Booth et al., 1967) or concomitant administration of AQ with other antimalarial drugs expressing similar profile such as sulfadoxine/pyrimethamine (SP) or proguanil (CDC, 1986) could be a problem.

In the current study we evaluated a single full course of AQ as opposed to repeated therapeutic courses to be adopted during preventive intermittent treatment. Because susceptibility of toxic serious adverse effects depends crucially on frequency intake, in this context, it is reasonable to speculate that the scheduled administration with spaced interval (e.g. 60 days during the planned study) preventive intermittent treatment will present no risks as a result of accumulation or carry-over effect of the drug. This assumption is based on clear evidence from pharmacokinetic studies indicating that the terminal elimination half-life of AQ is not longer than three weeks (Krishna \& White, 1996). Although the risks of serious toxic effects are considered to be much lower in therapeutic dosage, careful monitoring is important as the possibilities of toxicity in clinical practices cannot be excluded.

Amodiaquine achieved $100 \%$ parasitological clearance by day 7 . These results were not unexpected, as AQ has demonstrated to be superior to CQ and SP in the study area for treating uncomplicated malaria (MoH, 2000). The parasitological efficacy of AQ in asymptomatic volunteers in this study is of prospective importance for preventive intermittent treatment strategy, because it is likely that population harbouring malaria parasite, will have their parasitaemia cleared and subsequently protected from malaria clinical at- tack. Furthermore, data from chemotherapeutic studies of uncomplicated malaria indicate that treatment with an effective antimalarial drug results in significant haematocrit recovery (Nevill et al., 1994). In the present study a marginal gain in PCV values was observed. Malaria positive volunteers in both drugs gained much more than the aparasitaemic and the improvement was slightly pronounced in volunteers receiving AQ. This phenomenon provides more beneficial support of using $\mathrm{AQ}$ in preventive intermittent treatment strategy.

In conclusion the results from our study demonstrate that in chemotherapeutic dosage AQ did not induce serious life-threatening haematological adverse effects or liver toxicity; it was tolerable and efficacious against $P$. falciparum supporting the idea of using AQ for preventive intermittent treatment strategy.

\section{Acknowledgements}

The authors express their appreciation for the positive and co-operative role played by the volunteers, the administration and all member staff of Bombo Tanga Regional Hospital. We are particularly grateful to the excellent technical assistance of Mrs. Zaina Ali and Mr. Frank Magogo for laboratory work, Mr. Charles Kijazi and Ms. Asha Banda for their help with patient care. The study was supported in part by Health Users Trust Fund, (HUTF) Grant and DANIDA under ENRECA programme No. 104.Dan.8.1/402.

Received 10 April 2008

Revised 5 June 2008

Accepted 6 June 2008

\section{References}

Booth, K., Larkin, K., \& Maddocks, I. (1967) Agranulocytosis coincident with amodiaquine therapy. British Medical Journal 3, 32-33.

Brasseur, P., Guiguemde, R., Diallo, S., Guiyedi, V., Kombila, M., Ringwald, P. \& Olliaro, P. (1999) Amodiaquine remains effective for treating uncomplicated malaria in west and Central Africa. Transactions of the Royal Society of Tropical Medicine and Hygiene 93, 645-650.

Breckenridge, A.M. \& Winstanley, P.A. (1997) Clinical pharmacology and malaria Annals of Tropical Medicine and Parasitology 91, 727-733.

Greenwood, D. (1995) Conflicts of interest: the genesis of synthetic antimalarial agents in peace and war. Journal of Antimicrobial Chemotherapy 36, 857-872. 
CDC (1986) International notes agranulocytosis associated with the use of amodiaquine for malaria prophylaxis. Morbidity and Mortality Weekly Report 35, 165-166.

Hatton, C.S.R., Peto, T.E.A., Bunch, C., Pasvol, G., Russell, S.J., Singer, C.R., Edwards, G., \& Winstanley, P. (1986) Frequency of severe neutropenia associated with amodiaquine prophylaxis against malaria. Lancet 1, 411-414.

Kennedy, A.F. (1955) Absolute neutrophil leucopenia after uncontrolled use of amodiaquine. British Medical Journal ii, 475-476.

Krishna, S. \& White, N.J. (1996) Pharmacokinetics of quinine, chloroquine and amodiaquine. Clinical implications. Clinical Pharmacokinetic 30, 263-299.

Larrey, D., Castot, A., Pessayre, D., Merigot, P., Machayekhy, J.P, Feldmann, G., Lenoir, A., Rueff, B. \& Benhamou, J.P. (1968) Amodiaquine-induced hepatitis. A report of seven cases. Annals of Internal Medicine 104, 801-803.

Massaga, J.J., Kitua, A.Y., Lemnge, M.M., Akida, J.A., Malle, L.N., Ronn, A.M., Theander, T.G. \& Bygbjerg, I.C. (2003) Effect of intermittent treatment with amodiaquine on anemia and malarial fevers in infants in Tanzania: a randomized placebo-controlled trial. Lancet 361, 1853-1860.

Mengesha, T. \& Makonnen, E. (1999) Comparative efficacy and safety of chloroquine and alternative drugs: A Meta-analysis from six African countries. East African Medical Journal 76, 314-319.

MoH (2000) Implementation of a new antimalarial treatment policy in Tanzania: The rationale for change and guide to the process of policy implementation. Tanzania Health Research Bulletin 2, 10-13.
Nevill, C.G., Verhoefe, F.H., Munafu, C.G., Tenhove, W.R., Van Derkaay, H.J. \& Were J.B.O. (1994) A comparison of amodiaquine and chloroquine in the treatment therapy of falciparum malaria in Kenya. East African Medical Journal 71, 167-170.

Olliaro, P. Nevill, C. Le Bras, J., Ringwald, P., Massano, P., Garner, P., \& Brasseur, P. (1996) Systematic review of amodiaquine treatment in uncomplicated malaria. Lancet 348, 1196-1201.

Orrell, C., Taylor, W.R. \& Olliaro, P. (2001) Acute asymptomatic hepatitis in a healthy normal volunteer exposed to 2 oral doses of amodiaquine and artesunate. Transactions of the Royal Society of Tropical Medicine and Hygiene 95, 517-518.

Phillips-Howard, P.A. \& West, L.J. (1990) Serious adverse reactions to pyrimethamine-sulphadoxine, pyrimethamine - dapsone and to amodiaquine in Britain. Journal of the Royal Society of Medicine 83, 82-85.

Silverthorn, D.U. (1998) Human Physiology: an Integrated Approach. Prentice Hall, New Jersey, p. 478.

Winstanley, P.A., Simooya, O., Kofi-Ekue, J.M., Walker, O., Salako, L.A., Edward, G., Orme, M.E. \&Breckenridge, A.M. (1990) The disposition of amodiaquine in Zambians and Nigerians with malaria. British Journal of Clinical Pharmacology 29, 695-702.

WHO (1990) Practical Chemotherapy of Malaria. Report of WHO Scientific Group. Technical Report Series No. 805.

WHO (1998) Iron supplementation: large-scale trials to be supported, TDR News; No. 55, 4. 